\title{
Reflection Full Waveform Inversion with Decoupled Elastic-wave Equations in Inhomogeneous Medium
}

\author{
Zhanyuan Liang ${ }^{1}$, Guochen Wu*,2, Xiaoyu Zhang ${ }^{3}$, Qingyang $\mathrm{Li}^{4}$ \\ (1) China University of Petroleum Huadong - Qingdao Campus. Qingdao, CN 266555, e-mail: lzy09017216@163.com \\ (2) China University of Petroleum Huadong - Qingdao Campus. Qingdao, CN 266555, e-mail: guochenwu_wgc@163.com \\ (3) China University of Petroleum Huadong - Qingdao Campus. Qingdao, CN 266555, e-mail: 604810647@qq.com \\ (4) China University of Petroleum Huadong - Qingdao Campus. Qingdao, CN 266555, e-mail: $986132055 @ q q . c o m$
}

Article history: received September 21, 2019; accepted December 30, 2020

\begin{abstract}
Reflection full-waveform inversion (RFWI) can reduce the nonlinearity of inversion providing an accurate initial velocity model for full-waveform inversion (FWI) through the tomographic components (low-wavenumber). However, elastic-wave reflection full-waveform inversion (ERFWI) is more vulnerable to the problem of local minimum due to the complicated multi-component wavefield. Our algorithm first divides kernels of ERFWI into four subkernels based on the theory of decoupled elastic-wave equations. Then we try to construct the tomographic components of ERFWI with only single-component wavefields, similarly to acoustic inversions. However, the S-wave velocity is still vulnerable to the coupling effects because of $\mathrm{P}$-wave components contained in the $\mathrm{S}$-wavefield in an inhomogeneous medium. Therefore we develop a method for decoupling elasticwave equations in an inhomogeneous medium, which is applied to the decomposition of kernels in ERFWI. The new decoupled system can improve the accuracy of S-wavefield and hence further reduces the high-wavenumber crosstalk in the subkernel of S-wave velocity after kernels are decomposed. The numerical examples of Sigsbee2A model demonstrate that our ERFWI method with decoupled elastic-wave equations can efficiently recover the low-wavenumber components of the model and improve the precision of the S-wave velocity.
\end{abstract}

Keywords: Reflection full waveform inversion; Decoupled elastic-wave equations; Inhomogeneous medium; Kernels decomposition; Wave propagation.

\section{Introduction}

The traditional theory of velocity recovery is divided into two parts: background velocity construction and amplitude projection. In the first part, the background velocity model is built with methods such as traveltime tomography (including ray-based or wave-based, first-arrival traveltime tomography and reflection traveltime tomography) and velocity analysis. The high-wavenumber velocity is recovered using different migration 


\section{Zhanyuan Liang et al.}

methods [Claerbout and Doherty, 1972; Gazdag, 1978; Stolt, 1978; Baysal et al., 1983; Yilmaz, 2001; Biondi and Symes, 2004]. The problem of the traditional theory is its difficulty to update the velocity of intermediate wavelengths [Jannane et al., 1989]. In order to update velocity of different scales of wavelength simultaneously, Lailly [1983] and Tarantola [1984] proposed the full waveform inversion (FWI) theory that matches all the wavefield information without any approximation between the calculated data and the observed data in model parameter inversion. However in practice, this method is highly non-linear, thus suffering from many local minima (cycle-skipping) [Bunks et al., 1995].

To address the local minima issue, there are two strategies. The first strategy is inversion using the lowfrequency information in seismic data and the second strategy is inversion starting with an accurate initial model. The long-wavelength background velocity coverage with low-frequency data [Sirgue and Pratt, 2004] can reduce the dependence of FWI on the initial model. Bunks et al. [1995] proposed the multi-scale full waveform inversion method to combine the frequency of data with the scale of velocity. The multi-scale FWI method divides seismic data into different frequency and adopts the inverted results of low-frequency data as the initial velocity of the inversion of higher-frequency data. If there are sufficient low-frequency data, the multi-scale method can alleviate effects of an inaccuracy initial velocity. In general, low-frequency sources are not always available and the seismic data can go down only to approximately $5 \mathrm{~Hz}$ [Baeten et al., 2013; Shin and Cha, 2008; Wu et al., 2014]. So, if an accurate initial velocity model is crucial to FWI, more and more research is focusing on how to construct a good initial model and how to reduce the nonlinearity of FWI [Biondi and Almomin, 2013a; Biondi and Almomin, 2013b; Hardy, 2013; Djebbi, 2014].

According to the equation $\mathbf{k}=\left(2 f / c_{0}\right) \cos (\theta / 2) \mathbf{n}$ which is derived from the ray and Born approximate scattering theory [Virieux and Operto, 2009], where $c_{0}$ is the reference velocity, $\theta$ is the double of the incidence angle, the wavenumber $\mathbf{k}$ is proportional to the frequency $f$ and $\mathbf{n}$ is the direction vector of $\mathbf{k}$, the longwavelength background velocity may correspond to the data near zero frequency. Wu et al. [2014] simulated the pseudo-low-frequency data by Hilbert transformation and proposed the envelope inversion method. The frequency of envelope data is independent of the seismic frequency band, focusing around zero frequency. Similarly, Hu [2014] proposed beat tone inversion using two adjacent high frequencies to obtain a good initial model. Bharadwaj et al. [2016] proposed a bump data function to obtain the long-wavelength structure.

Alternatively, on the basis of the equation $\mathbf{k}=\left(2 f / c_{0}\right) \cos (\theta / 2) \mathbf{n}$, fixing the frequency $f$ of seismic data, the wavenumber $\mathbf{k}$ decreases as $\theta$ (from $0^{\circ}$ to $180^{\circ}$ ) increases. So wave components with large scattering angles such as direct waves, diving waves and refraction waves mainly update the background velocity of shallow layers, while reflection waves with smaller scattering angles mainly recover the perturbation velocity of high wavenumbers in the middle and deep layers. Accordingly, Mora [1989] divided the gradient of FWI into migration components and tomographic components corresponding to high-wavenumber and low-wavenumber respectively. However, the energy of migration components is far stronger than that of tomographic components [Tang et al., 2013]. Therefore, FWI mainly just updates the high-wavenumber velocity. With respect to reflection data, the (gradient) model update of FWI is similar to a reverse time migration (RTM) image of the data residual [Wang et al., 2018b]. Therefore, Díaz and Sava [2014] adopted a directional filter based on Poynting vectors in the gradient computation to preserve the smooth components of the gradient, thereby spreading information away from the sharp boundary. However, as the initial velocity model of FWI is smooth, there are only the downgoing waves in the wavefield rather than the upgoing waves. To address this issue, Xu et al. [2012a, 2012b] proposed a reflection FWI (RFWI) method with a migration/demigration [Symes and Kern, 1994] process to generate the upgoing waves and recover the background velocity along the tomographic components. For the issue that RFWI with the least square objective function of amplitude can easily fall into local minima, Chi et al. [2015] developed a correlation-based RFWI method with better convexity of the objective function.

Over the past few years, most geophysicists have focused on the development of acoustic FWI methods, based on the assumption of acoustic medium only. However, S-waves also play an important role in the illumination of targets under a gas cloud, salt body and fractures, motivating the development from singleparameter acoustic-wave FWI to multi-parameter elastic-wave FWI (EFWI) [Tarantola, 1986; Mora, 1987; Crase et al., 1990, Wang et al., 2017]. However, between the inversion of multiple parameters trade-off is inevitable, which increases the nonlinearity of inversion. Wang et al. [2018b] decomposed kernels of gradient by decoupling elastic-wave equations to update the P-wave velocity and S-wave velocity separately like the acoustic case. In addition, the update for the S-wave velocity in FWI intrinsically brings even higher wavenumbers than P-waves 
because of the lower velocity of S-waves. Thus, optimizing the low-wavenumber components for the S-wave velocity is even more crucial in preventing the elastic FWI from converging to local minima [Wang et al., 2018a].

Methods of elastic-wave equation decoupling are generally divided into two categories. The first category of methods uses Helmholtz decomposition for elastic wavefield separation [e.g., Morse and Feshbach, 1953; Clayton, 1981; Mora, 1987]. These methods not only increase the numerical complexity and computational cost of the elastic-wave simulation, but also affect the amplitude and phase of the wave field extrapolation [Chang and McMechan, 1987; Sun and McMechan, 2001; Sun et al., 2011; Du et al., 2012, 2014; Wang and McMechan, 2015; Cheng et al., 2016]. The second category of decoupling method starts from a set of decoupling equations which maintain the vector characteristics and elastic characteristics of the seismic wave propagation. Ma and Zhu [2003] decoupled the P- and S-wave equations similarly to wavefield extrapolation by defining the P-wave stress. Xiao and Leaney [2010] used different but equivalent equations to decompose the elastic-wave field for RTM of vertical seismic profiling data. Subsequently, several similar ideas were proposed to separate the P- and S-waves for imaging and migration velocity analysis [Shabelansky et al., 2015; Wang and McMechan, 2015; Du et al., 2017].

In this paper, we propose a set of decoupled elastic-wave equations in an inhomogeneous medium to reduce the crosstalk artifact in the elastic-wave reflection full waveform inversion (ERFWI) after kernels are decomposed. We first introduce kernels of RFWI based on the first-order Born approximation. Accordingly, kernel of ERFWI is divided into four subkernels based on the method of Wang et al. [2018b]. After kernels are decomposed, the ERFWI of S-wave velocity can be recovered with only S-wavefield, similarly to acoustic RFWI. The problem is that ERFWI begins with an initial velocity model which is actually for an inhomogeneous medium. Hence, we derive a set of equations to address the decoupling in an inhomogeneous medium to obtain the S-wavefield with less P-wave components. Then we test the new decoupled method with a simple inhomogeneous model, showing that it can enhance the accuracy of S-wavefield and it can further improve subkernels of ERFWI. In the numerical example session, we apply ERFWI with the conjugation gradient method to the Sigsbee2A model to demonstrate the effectiveness of our proposed method.

\section{Methods}

\subsection{Kernels of Reflection Full Waveform Inversion}

The purpose of reflection full waveform (RFWI) inversion is to update low-wavenumber velocity along to the "rabbit-ear" path [Xu et al., 2012]. The key of RFWI is to calculate the contribution of background velocity perturbation to wavefield perturbation. So, we firstly decompose the velocity into background component and perturbation component in the process of kernels derivation. Correspondingly, the wavefield is also decomposed into background and perturbation.

Decomposition of model parameters into background $\mathbf{m}_{0}(\mathbf{x})$ and perturbation $\delta \mathbf{m}(\mathbf{x})$ is as below:

$$
\mathbf{m}(\mathbf{x})=\mathbf{m}_{0}(\mathbf{x})+\delta \mathbf{m}(\mathbf{x}) .
$$

Similarly, wavefield $\mathbf{u}(\mathbf{x}, t)$ can be decomposed into background $\mathbf{u}_{0}(\mathbf{x}, t)$ and perturbation $\delta \mathbf{u}(\mathbf{x}, t)$ :

$$
\mathbf{u}(\mathbf{x}, t)=\mathbf{u}_{0}(\mathbf{x}, t)+\delta \mathbf{u}(\mathbf{x}, t)
$$

According to the first-order Born approximation, the scattering wavefield is far weaker than the incident wavefield, i.e. $\delta \mathbf{u}(\mathbf{x}, t)=\mathbf{u}(\mathbf{x}, t)$. Meanwhile, we express the integral solution of the perturbation wavefield in the frequency domain in order to void the derivative calculation [Xu et al., 2012]:

$$
\delta \mathbf{u}\left(\mathbf{x}, \omega ; \mathbf{x}_{S}\right)=\omega^{2} f(\omega) \int \delta \mathbf{m}\left(\mathbf{x}^{\prime}\right) \mathbf{G}_{0}\left(\mathbf{x}, \omega ; \mathbf{x}^{\prime}\right) \mathbf{G}_{0}\left(\mathbf{x}^{\prime}, \omega ; \mathbf{x}_{S}\right) d \mathbf{x}^{\prime},
$$

where $\omega$ represents frequency, $f(\omega)$ is the source signature, $\mathbf{G}_{0}\left(\mathbf{x}, \omega ; \mathbf{x}^{\prime}\right)$ and $\mathbf{G}_{0}\left(\mathbf{x}^{\prime}, \omega ; \mathbf{x}_{s}\right)$ are Green's function of source wavefield and receiver wavefield respectively. $\mathbf{G}_{0}$ means the Green's function for the background velocity and in $\left(\mathbf{x}^{\prime}, \omega ; \mathbf{x}_{s}\right), \mathbf{x}^{\prime}$ is the scattered point and $\mathbf{x}_{s}$ is the source position. According to the reciprocity of Green's function, $\mathbf{x}$ 


\section{Zhanyuan Liang et al.}

and $\mathbf{y}$ can be exchanged:

$$
\frac{\partial \delta \mathbf{u}\left(\mathbf{y}, \omega ; \mathbf{x}_{s}\right)}{\partial \mathbf{m}_{0}}=\omega^{2} f(\omega) \int\left(\begin{array}{c}
\delta \mathbf{m}\left(\mathbf{x}^{\prime}\right) \frac{\partial \mathbf{G}_{0}\left(\mathbf{x}^{\prime}, \omega ; \mathbf{x}_{s}\right)}{\left.\partial \mathbf{m}_{0}\right)} \mathbf{G}_{0}\left(\mathbf{x}^{\prime}, \omega ; \mathbf{y}\right) \\
+\delta \mathbf{m}\left(\mathbf{x}^{\prime}\right) \frac{\partial \mathbf{G}_{0}\left(\mathbf{x}^{\prime}, \omega ; \mathbf{y}\right)}{\partial \mathbf{m}_{0}} \mathbf{G}_{0}\left(\mathbf{x}^{\prime}, \omega ; \mathbf{x}_{S}\right)
\end{array}\right) d \mathbf{x}^{\prime}
$$

where,

$$
\frac{\partial \mathbf{G}_{0}\left(\mathbf{x}^{\prime}, \omega ; \mathbf{x}_{\mathbf{s}}\right)}{\partial \mathbf{m}_{0}}=\omega^{2} \mathbf{G}_{0}\left(\mathbf{x}^{\prime}, \omega ; \mathbf{x}\right) \mathbf{G}_{0}\left(\mathbf{x}, \omega ; \mathbf{x}_{S}\right) .
$$

Substitute Equation 5 into Equation 4:

$$
\frac{\partial \delta \mathbf{u}\left(\mathbf{y}, \omega ; \mathbf{x}_{s}\right)}{\partial \mathbf{m}_{0}}=\omega^{2} f(\omega) \int\left(\begin{array}{c}
\omega^{2} \delta \mathbf{m}\left(\mathbf{x}^{\prime}\right) \mathbf{G}_{0}\left(\mathbf{x}^{\prime}, \omega ; \mathbf{x}\right) \mathbf{G}_{0}\left(\mathbf{x}, \omega ; \mathbf{x}_{s}\right) \mathbf{G}_{0}\left(\mathbf{x}^{\prime}, \omega ; \mathbf{y}\right) \\
+\omega^{2} \delta \mathbf{m}\left(\mathbf{x}^{\prime}\right) \mathbf{G}_{0}\left(\mathbf{x}^{\prime}, \omega ; \mathbf{x}\right) \mathbf{G}_{0}(\mathbf{x}, \omega ; \mathbf{y}) \mathbf{G}_{0}\left(\mathbf{x}^{\prime}, \omega ; \mathbf{x}_{s}\right)
\end{array}\right) d \mathbf{x}^{\prime}
$$

The Green's function of first-order scattering wavefield can be expressed as:

$$
\mathbf{G}_{s}(\mathbf{x}, \omega ; \mathbf{y})=\int \omega^{2} \delta \mathbf{m}\left(\mathbf{x}^{\prime}\right) \mathbf{G}_{0}\left(\mathbf{x}, \omega ; \mathbf{x}^{\prime}\right) \mathbf{G}_{0}\left(\mathbf{x}^{\prime}, \omega ; \mathbf{y}\right) d \mathbf{x}^{\prime}
$$

When $\mathbf{y}=\mathbf{x}_{g}, \mathbf{x}_{g}$ is the location of geophone, we can get:

$$
\frac{\partial \delta \mathbf{u}\left(\mathbf{x}_{g}, \omega ; \mathbf{x}_{s}\right)}{\partial \mathbf{m}_{0}}=\omega^{2} f(\omega) \mathbf{G}_{0}\left(\mathbf{x}, \omega ; \mathbf{x}_{S}\right) \mathbf{G}_{s}\left(\mathbf{x}, \omega ; \mathbf{x}_{g}\right)+\mathbf{G}_{s}\left(\mathbf{x}, \omega ; \mathbf{x}_{S}\right) \mathbf{G}_{0}\left(\mathbf{x}, \omega ; \mathbf{x}_{g}\right) .
$$

The Equation 8 is the kernel of RFWI which represents the contribution of wavefield perturbation corresponding to the background model parameters perturbation.

\subsection{Elastic-wave Reflection Full Waveform Inversion and Kernels decomposed}

The objective function of ERFWI is established as below:

$$
O_{E R F W I}\left(\mathbf{m}_{0}\right)=\frac{1}{2} \sum_{s, g} \int\left|u_{s c a l}\left(\mathbf{x}_{g}, t ; \mathbf{x}_{s}\right)-u_{o b s}\left(\mathbf{x}_{g}, t ; \mathbf{x}_{s}\right)\right| d t,
$$

where $u_{\text {scal }}\left(\mathbf{x}_{g}, t ; \mathbf{x}_{s}\right)$ represents the calculated scattering wavefield at the location of receivers, while $u_{o b s}\left(\mathbf{x}_{g}, t ; \mathbf{x}_{s}\right)$ represents the observed wavefield. The gradient of ERFWI is the derivative of the objective function with respect to background model parameters,

$$
\frac{\partial O_{E R F W I}\left(\mathbf{m}_{0}\right)}{\partial \mathbf{m}_{0}}=\sum_{s, g} \int \frac{\partial u_{s}\left(\mathbf{x}_{g}, t ; \mathbf{x}_{s}\right)}{\partial \mathbf{m}_{0}}\left[u_{s c a l}\left(\mathbf{x}_{g}, t ; \mathbf{x}_{s}\right)-u_{o b s}\left(\mathbf{x}_{g}, t ; \mathbf{x}_{s}\right)\right] d t .
$$

Substituting equation 8 into equation 10, we get the gradient expression of ERFWI which is the summation of the cross-correlation between the source-side background wavefield and the receiver-side scattered wavefield, and the cross-correlation between the source-side scattered wavefield and the receiver-side background wavefield. For simplicity, equation 10 can be expressed as:

$$
\nabla O_{E R F W I}\left(\mathbf{m}_{0}\right)=-\int(\boldsymbol{\varphi} \cdot \delta \boldsymbol{\Psi}+\delta \boldsymbol{\varphi} \cdot \boldsymbol{\Psi})
$$

where $\varphi$ represents the source-side background wavefield, $\Psi$ represents the receiver-side background wavefield, $\delta \varphi$ 
represents the source-side scattered wavefield, and $\delta \Psi$ represents the receiver-side scattered wavefield.

As we know, there is only PP wave mode in the acoustic RFWI and the inverted parameter is only $V_{P}$, so no crosstalk exists. However, due to the co-existence of P- and S-waves, ERFWI suffers from crosstalk noise, especially for the S-wave velocity [Wang et al., 2018b]. Under the assumption of homogeneous medium, the elastic wavefield can be completed decoupled into P-wave wavefield and S-wave wavefield [Ma and Zhu, 2003; Xiao and Leaney, 2010]. According to the decoupled wavefield, we decompose the gradient of ERFWI into four parts:

$$
\begin{aligned}
& K^{P P}=-\int\left(\boldsymbol{\varphi}_{P} \cdot \delta \boldsymbol{\Psi}_{P}+\delta \boldsymbol{\varphi}_{P} \cdot \boldsymbol{\Psi}_{P}\right) \\
& K^{P S}=-\int\left(\boldsymbol{\varphi}_{P} \cdot \delta \boldsymbol{\Psi}_{S}+\delta \boldsymbol{\varphi}_{P} \cdot \boldsymbol{\Psi}_{S}\right) \\
& K^{S P}=-\int\left(\boldsymbol{\varphi}_{S} \cdot \delta \boldsymbol{\Psi}_{P}+\delta \boldsymbol{\varphi}_{S} \cdot \boldsymbol{\Psi}_{P}\right) \\
& K^{S S}=-\int\left(\boldsymbol{\varphi}_{S} \cdot \delta \boldsymbol{\Psi}_{S}+\delta \boldsymbol{\varphi}_{S} \cdot \boldsymbol{\Psi}_{S}\right)
\end{aligned}
$$

where:

(i) $K^{P P}$ is the subkernel through the summation of the cross-correlation between the source-side P-wave background wavefield and the receiver-side P-wave scattered wavefield, and the cross-correlation between the source-side P-wave scattered wavefield and the receiver-side P-wave background wavefield;

(ii) $K^{P S}$ is the subkernel through the summation of the cross-correlation between the source-side P-wave background wavefield and the receiver-side S-wave scattered wavefield, and the cross-correlation between the source-side P-wave scattered wavefield and the receiver-side S-wave background wavefield;

(iii) $K^{S P}$ is the subkernel through the summation of the cross-correlation between the source-side S-wave background wavefield and the receiver-side P-wave scattered wavefield, and the cross-correlation between the source-side S-wave scattered wavefield and the receiver-side P-wave background wavefield;

(iv) $K^{S S}$ is the subkernel through the summation of the cross-correlation between the source-side S-wave background wavefield and the receiver-side S-wave scattered wavefield, and the cross-correlation between the source-side S-wave scattered wavefield and the receiver-side S-wave background wavefield.

Here we assume the density is constant, so that inversion of just P-wave velocity and S-wave velocity is needed, and inversion effects of the two model parameters will be considered. Firstly, we assume $\delta V_{P} \neq 0, \delta V_{S} \neq 0$ and hence simply invert the P-wave velocity without the S-wave velocity. According to the radiation pattern (Figure 1), we can see there is only PP scattered wave, without PS converted wave. So in this case, we need to consider only the subkernel $K^{P P}$ in the gradient of ERFWI (Figure 2), similarly as in the acoustic RFWI.

\begin{tabular}{|l|c|c|}
\hline & $\delta V_{P} \rightarrow P P$ & $\delta V_{P} \rightarrow P S$ \\
\hline$\delta V_{P} \neq 0$ & $\delta V_{S}=0$ &
\end{tabular}

Figure 1. Radiation pattern of $V_{P}$ perturbation with P-wave incidence. 


\section{Zhanyuan Liang et al.}

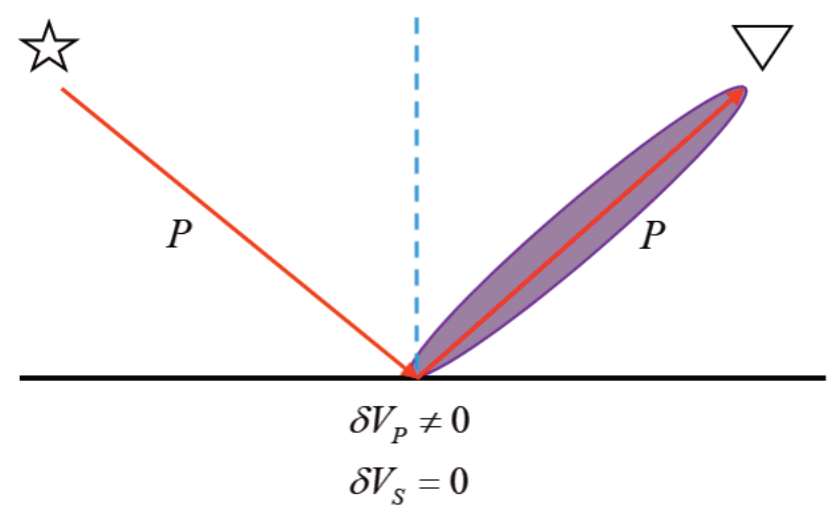

Figure 2. A schematic of ERFWI with only $V_{P}$ inversion (receiver-side).

Next we assume $\delta V_{P}=0, \delta V_{S} \neq 0$, meaning only S-wave velocity will be inverted instead of P-wave velocity. In this case, P-wave and S-wave exist simultaneously in the wavefield (Figure 3) and all the four subkernels $K^{P P}, K^{P S}, K^{S P}$, $K^{S S}$ in the gradient of ERFWI together cause the "cross-talk" due to the superposition of different wave paths. As shown in Figure 4, which takes the wave path of receiver-side as an example, the subkernel shown by the green ellipse area can be contaminated by the subkernel shown by the purple ellipse area. The subkernel with converted waves may bring many high-wavenumber information which undermines the inversion of the low-wavenumber velocity.

\begin{tabular}{|l|l|l|}
\hline$\delta V_{S} \rightarrow P P$ & $\delta V_{S} \rightarrow P S$ \\
\hline$\delta V_{P}=0$ \\
$\delta V_{S} \neq 0$
\end{tabular}

Figure 3. Radiation pattern of $V_{P}$ perturbation with P-wave incidence.

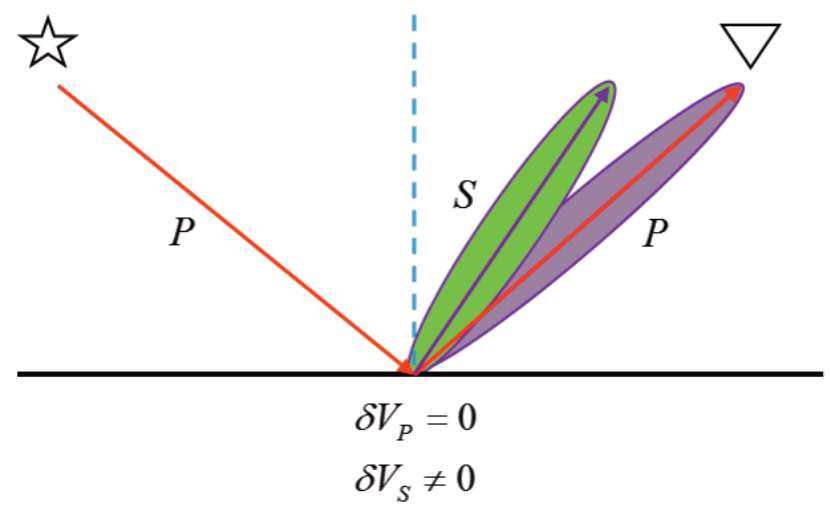

Figure 4. A schematic of ERFWI with only $V_{P}$ inversion (receiver-side). 


\section{Decoupled ERWI in Inhomogeneous Medium}

The method of decoupled elastic-wave equations can reduce the crosstalk problem of S-wave velocity inversion in ERFWI [Wang et al., 2018a; Wang et al., 2018b]. The decoupled method can decompose the elastic wavefield into a P-wavefield and S-wavefield, which are then inverted separately.

We establish a velocity model with $\delta V_{P}=0, \delta V_{S} \neq 0$ to invert only $V_{S}$. The P-wave velocity of the model is homogenous and there is a layer to generate the scattered wave and converted wave in the deep area of the S-wave velocity model. By decomposition of the elastic wavefield into P-wavefield and S-wavefield, we can calculate subkernels separately as shown in Figure 5a, 5b, $5 c$ and $5 d$. From the Figure 5, we can see that Figure $5 b$ and $5 c$ mainly bring high-wavenumber noise to ERFWI and the Figure $5 \mathrm{~d}$ is beneficial to the update of low-wavenumber components. As shown in Figure 6, the decoupled kernel of $V_{S}$ contains only with $S$-S wavefield. Now, the kernel of $V_{S}$ can be expressed:

$$
K_{V_{S}}^{S S}=-\int \delta \boldsymbol{\varphi}_{S} \cdot \boldsymbol{\Psi}_{S}
$$
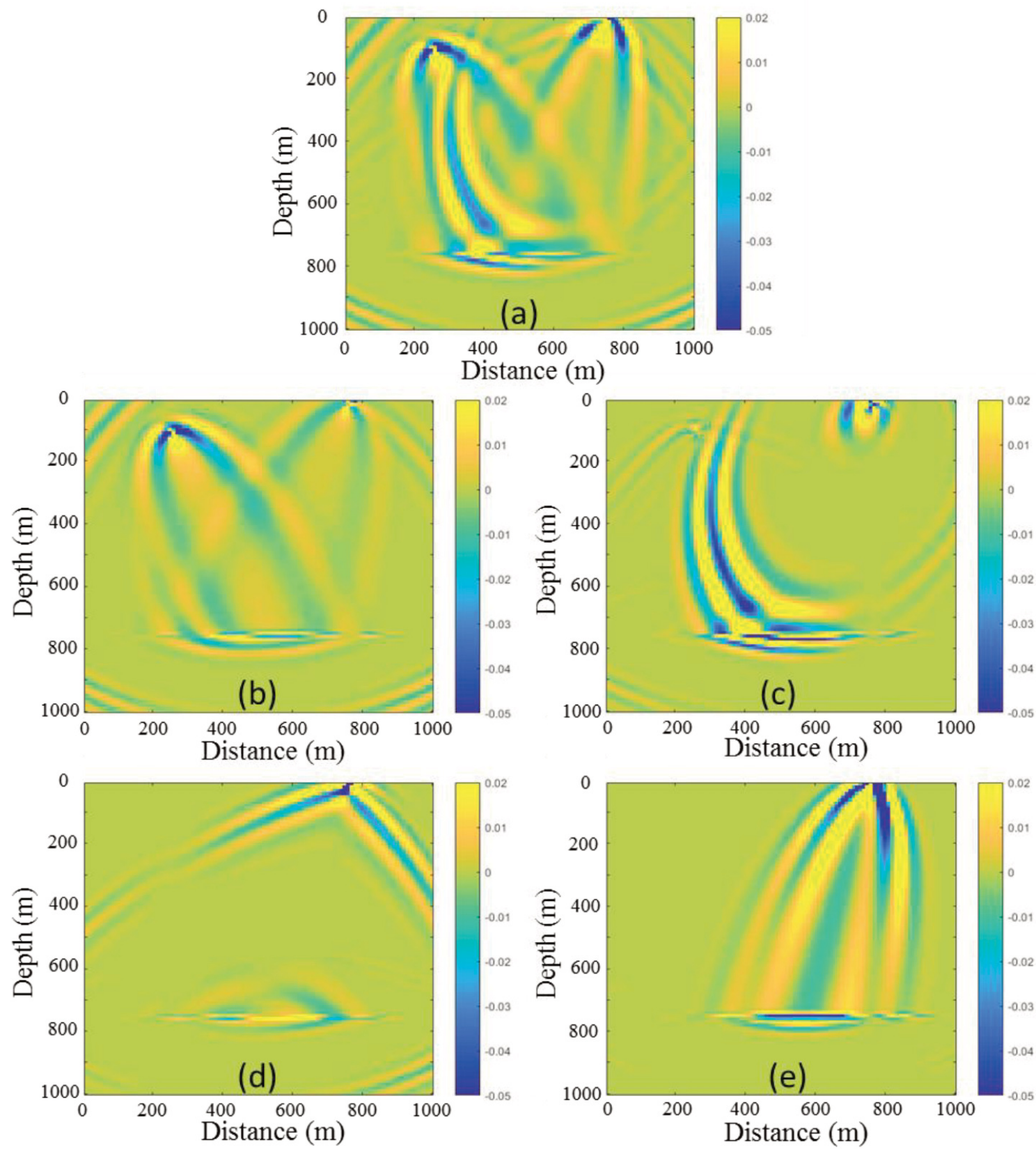

Figure 5. Kernels decomposed of $V_{P}$ of ERFWI. (a) Kernel of $V_{P}$ with the full wavefield, (b) Kernel of $V_{P}$ only with $P$ - $P$ wavefield, (c) Kernel of $V_{P}$ only with $P-S$ wavefield, (d) Kernel of $V_{P}$ only with $S$ - $P$ wavefield, (e) Kernel of $V_{P}$ only with $S$ - $S$ wavefield. 
Zhanyuan Liang et al.

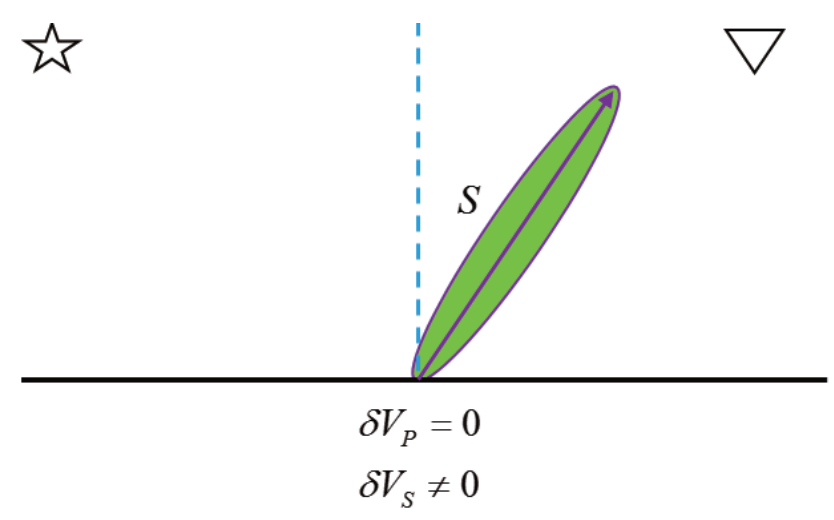

Figure 6. A schematic of $V_{P}$ inversion based on elastic wavefield vector decoupled (receiver-side).
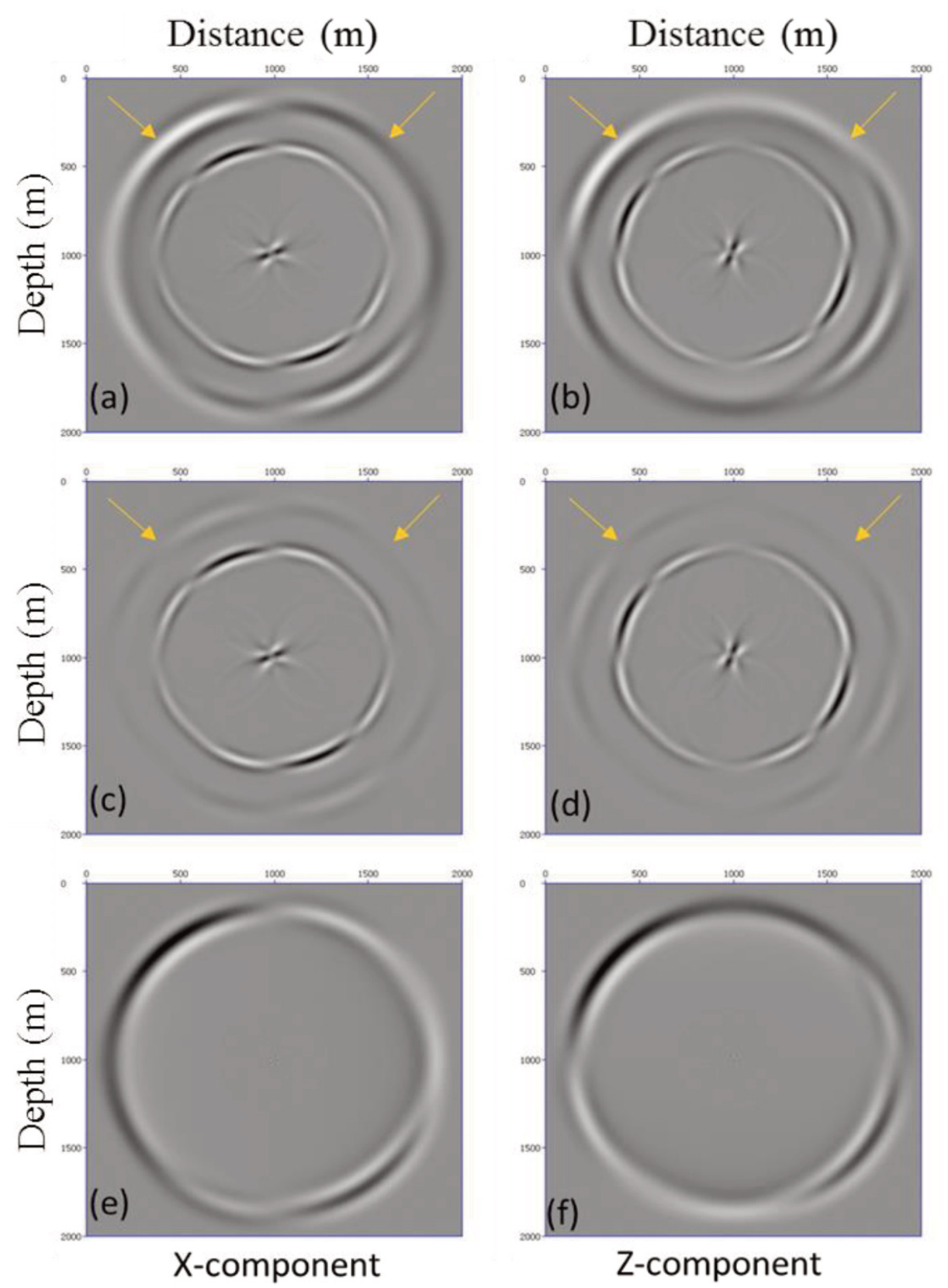

Figure 7. Snapshots of S-wavefield. (a) (b) The S-wavefield with inhomogeneous-term, (c) (d) S-wavefield without inhomogeneous-term, (e) (f) the difference between the above two methods (a) (c) (e) X-components of wavefield, (b) (d) (f) Z-components of wavefield. 


\section{Decoupled ERWI in Inhomogeneous Medium}

Notice that the P-wavefield and the S-wavefield can be completely decoupled only under the homogenous medium assumption. As a matter of fact, most models are inhomogeneous to some extent, which means homogenous decoupled theory is not sufficient to decompose inhomogeneous elastic wavefield. As a consequence, the decoupled S-wavefield will contain P-wavefield components, as shown in Figure $8 \mathrm{a}$ and $8 \mathrm{~b}$, so the $K_{V_{S}}^{S S}$ will be contaminated by other kernels. In this paper, we propose a set of elastic vector decoupled equations for inhomogeneous medium to improve the accuracy of S-wavefield for the ERFWI of low-wavenumber $V_{S}$.
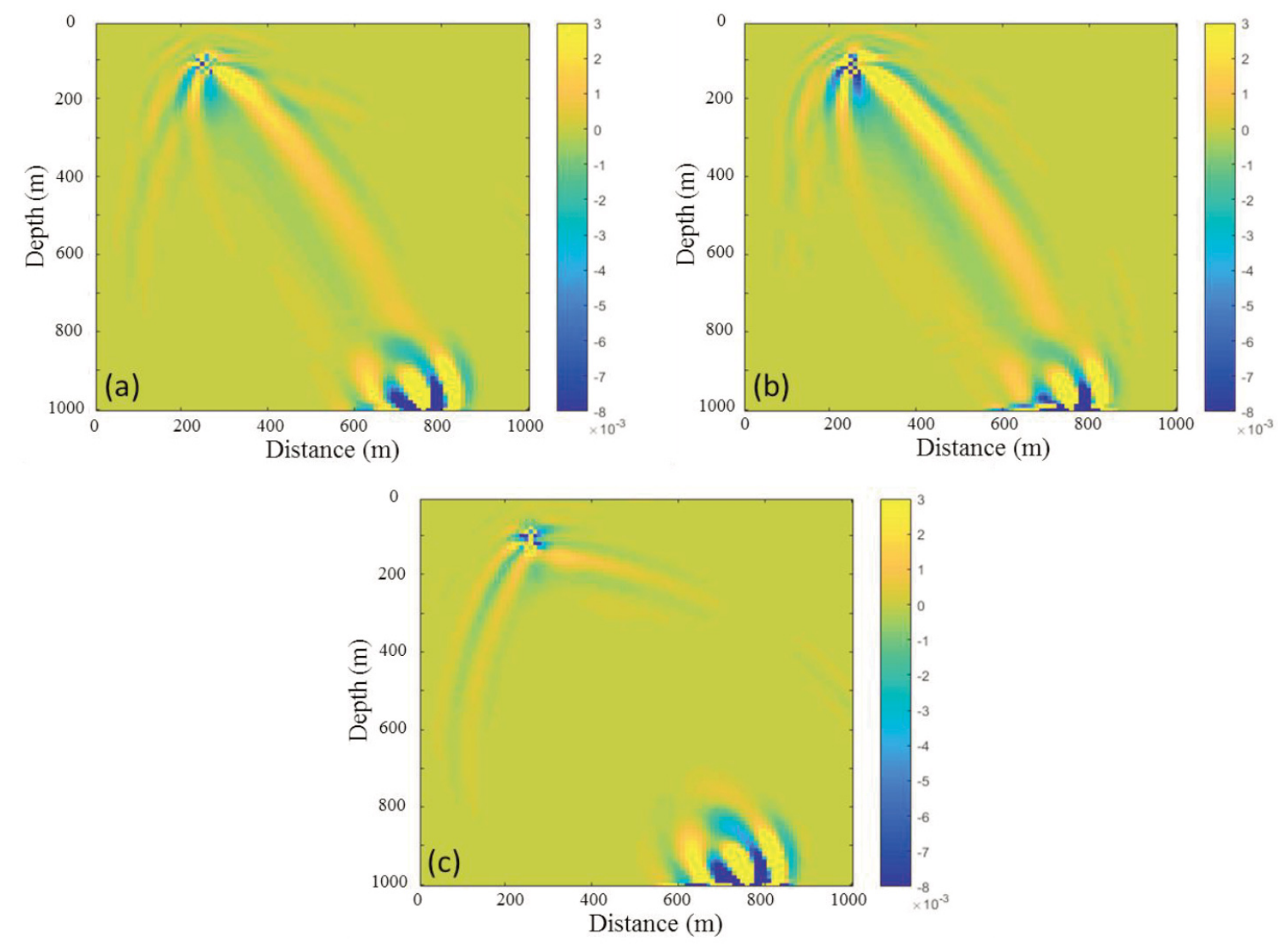

Figure 8. Kernels of S-wave velocity in inhomogeneous medium, $K_{V_{S}}^{S S}$ Kernel of S-wave velocity with conventional decoupled method (a), our decoupled method (b), and the difference (c) between the two methods.

\subsection{Elastic Vector Decoupled Equations in Inhomogeneous Medium}

The second-order elastic-wave equation in inhomogeneous medium is written with the form of matrix vector symbols as:

$$
\ddot{\mathbf{u}}=\left[\nabla\left(V_{P}^{2}-2 V_{S}^{2}\right)\right](\nabla \cdot \mathbf{u})+\nabla V_{S}^{2} \cdot\left[\nabla \mathbf{u}+(\nabla \mathbf{u})^{T}\right]+V_{P}^{2} \nabla \nabla \cdot \mathbf{u}-V_{S}^{2} \nabla \times \nabla \times \mathbf{u},
$$

where $\ddot{\mathbf{u}}=\partial^{2} u / \partial t^{2}$ is the vector wavefield of acceleration, $\nabla=\left[\partial_{i}, \partial_{j}, \partial_{k}\right]$ represents the gradient operator, $\nabla$. represents the divergence operator, $\nabla \times$ represents the curl operator, $\nabla \mathbf{u}=\partial u_{i} / \partial x_{j}$, and $(\nabla \mathbf{u})^{T}=\partial u_{j} / \partial x_{i}$.

Calculate the divergence of Equation 14 [Li et al., 2018]:

$$
\ddot{P}=P \nabla^{2} V_{P}^{2}+2 \nabla V_{P}^{2} \cdot \nabla P-2 P \nabla^{2} V_{S}^{2}-2 \nabla V_{S}^{2} \cdot \nabla \times S+V_{P}^{2} \nabla^{2} P
$$




\section{Zhanyuan Liang et al.}

where $P=\nabla \cdot \mathbf{u}$. Equation 15 implies the corresponding relation between the P-wave and the velocity perturbation: $\delta V_{P} \rightarrow P P$ (PP scattered wave by $V_{P}$ perturbation, the same below). $\delta V_{S} \rightarrow P P, \delta V_{S} \rightarrow S P$.

Take the calculation of the curl of Equation 14 [Li et al., 2018]:

$$
\ddot{\mathbf{S}}=\nabla V_{S}^{2} \cdot \nabla \mathbf{S}+\left(\nabla V_{S}^{2}\right) \times(\nabla \times \mathbf{S})+2\left(\nabla V_{S}^{2}\right) \times(\nabla P)+V_{S}^{2} \nabla^{2} \mathbf{S},
$$

where $\mathbf{S}=\nabla \times \mathbf{u}$. Equation 16 shows the corresponding relation between the S-wave and the velocity perturbation: $\delta V_{S} \rightarrow P P, \delta V_{S} \rightarrow P S$.

Based on Helmholtz's theory, we can decompose the elastic-wave wavefield into P- and S-wave wavefield as:

$$
\frac{\partial^{2} \mathbf{u}}{\partial t^{2}}=\frac{\partial^{2} \mathbf{u}_{P}}{\partial t^{2}}+\frac{\partial^{2} \mathbf{u}_{S}}{\partial t^{2}}=\nabla\left(V_{P}^{2} \nabla \cdot \mathbf{u}\right)-\nabla \times\left(V_{S}^{2} \nabla \times \mathbf{u}\right),
$$

where $\mathbf{u}_{P}=\left[u_{P} w_{P}\right]$ and $\mathbf{u}_{S}=\left[u_{S} w_{S}\right]$ represent P- and S-wave wavefield respectively. When model parameters are homogeneous, we can rewrite Equation 14 as:

$$
\ddot{\mathbf{u}}^{\prime}=V_{P}^{2} \nabla\left(\nabla \cdot \mathbf{u}^{\prime}\right)-V_{S}^{2} \nabla \times\left(\nabla \times \mathbf{u}^{\prime}\right),
$$

where $\mathbf{u}^{\prime}$ represents the vector of displacement wavefield in homogeneous medium. Equation 18 can be decoupled completely, with corresponding relation between the P-wave and the S-wave with the velocity perturbation: $\delta V_{S} \rightarrow P P, \delta V_{S} \rightarrow S S$. Comparing Equation 18 and Equation 14, we can conclude that P-wave and S-wave are coupled due to the $V_{S}$ perturbation just like the radiation pattern of $V_{S}$ (Figure 3). We use 2D displacement components to express Equation 18 as:

$$
\begin{aligned}
& \frac{\partial^{2} u^{\prime}}{\partial t^{2}}=V_{P}^{2} \frac{\partial}{\partial x}\left(\frac{\partial u^{\prime}}{\partial x}+\frac{\partial w^{\prime}}{\partial z}\right)+V_{S}^{2} \frac{\partial}{\partial z}\left(\frac{\partial u^{\prime}}{\partial z}-\frac{\partial w^{\prime}}{\partial x}\right) \\
& \frac{\partial^{2} w^{\prime}}{\partial t^{2}}=V_{P}^{2} \frac{\partial}{\partial z}\left(\frac{\partial u^{\prime}}{\partial x}+\frac{\partial w^{\prime}}{\partial z}\right)+V_{S}^{2} \frac{\partial}{\partial x}\left(\frac{\partial w^{\prime}}{\partial x}-\frac{\partial u^{\prime}}{\partial z}\right),
\end{aligned}
$$

where $u^{\prime}$ and $w^{\prime}$ represent the horizontal and vertical components of displacement wavefield respectively. Similarly, we use 2D displacement components to express Equation 14 as:

$$
\begin{aligned}
& \frac{\partial^{2} u}{\partial t^{2}}=\frac{\partial}{\partial x}\left[V_{P}^{2}\left(\frac{\partial u}{\partial x}+\frac{\partial w}{\partial z}\right)\right]+\frac{\partial}{\partial z}\left[V_{S}^{2}\left(\frac{\partial u}{\partial z}-\frac{\partial w}{\partial x}\right)\right]+2\left(\frac{\partial V_{S}^{2}}{\partial z} \frac{\partial w}{\partial x}-\frac{\partial V_{S}^{2}}{\partial x} \frac{\partial w}{\partial z}\right) \\
& \frac{\partial^{2} w}{\partial t^{2}}=\frac{\partial}{\partial z}\left[V_{P}^{2}\left(\frac{\partial u}{\partial x}+\frac{\partial w}{\partial z}\right)\right]+\frac{\partial}{\partial x}\left[V_{S}^{2}\left(\frac{\partial w}{\partial x}-\frac{\partial u}{\partial z}\right)\right]+2\left(\frac{\partial V_{S}^{2}}{\partial x} \frac{\partial w}{\partial z}-\frac{\partial V_{S}^{2}}{\partial z} \frac{\partial u}{\partial x}\right),
\end{aligned}
$$

where $u$ and $w$ represent the horizontal and vertical components of displacement wavefield respectively. Subtracting Equation 19 by Equation 20, we have [Tang and McMechan, 2018]:

$$
\begin{aligned}
& \frac{\partial^{2} u}{\partial t^{2}}-\frac{\partial^{2} u_{P}}{\partial t^{2}}-\frac{\partial^{2} u_{S}}{\partial t^{2}}=2\left(\frac{\partial V_{S}^{2}}{\partial z} \frac{\partial w}{\partial x}-\frac{\partial V_{S}^{2}}{\partial x} \frac{\partial w}{\partial z}\right) \\
& \frac{\partial^{2} w}{\partial t^{2}}-\frac{\partial^{2} w_{P}}{\partial t^{2}}-\frac{\partial^{2} w_{S}}{\partial t^{2}}=2\left(\frac{\partial V_{S}^{2}}{\partial x} \frac{\partial w}{\partial z}-\frac{\partial V_{S}^{2}}{\partial z} \frac{\partial u}{\partial x}\right),
\end{aligned}
$$

For convenience, we define the right terms of Equation 21 as "inhomogeneous-term" which contains the spatial derivative of S-wave velocity, i.e. $\partial V_{S}^{2} / \partial x$ and $\partial V_{S}^{2} / \partial z$. According to the relationship between wavefield and velocity perturbation in Equation 15, 16 and 18, we can get the contribution of the inhomogeneous-term to the wavefield: $\delta V_{S} \rightarrow P P, \delta V_{S} \rightarrow P S, \delta V_{S} \rightarrow S P$. Among them, the energy of PP scattered wave $\left(\delta V_{S} \rightarrow P P\right)$ is stronger than the 
converted wave PS $\left(\delta V_{S} \rightarrow P S\right)$ and SP $\left(\delta V_{S} \rightarrow S P\right)$. We define the new elastic-wave vector decoupled equations in inhomogeneous medium:

$$
\begin{gathered}
\frac{\partial^{2} u_{P}}{\partial t^{2}}=\frac{\partial}{\partial x}\left[V_{P}^{2}\left(\frac{\partial u}{\partial x}+\frac{\partial w}{\partial z}\right)\right]+2\left(\frac{\partial V_{S}^{2}}{\partial z} \frac{\partial w}{\partial x}-\frac{\partial V_{S}^{2}}{\partial x} \frac{\partial w}{\partial z}\right) \\
\frac{\partial^{2} w_{P}}{\partial t^{2}}=\frac{\partial}{\partial z}\left[V_{P}^{2}\left(\frac{\partial u}{\partial x}+\frac{\partial w}{\partial z}\right)\right]+2\left(\frac{\partial V_{S}^{2}}{\partial x} \frac{\partial u}{\partial z}-\frac{\partial V_{S}^{2}}{\partial z} \frac{\partial u}{\partial x}\right) \\
\frac{\partial^{2} u_{S}}{\partial t^{2}}=\frac{\partial}{\partial z}\left[V_{S}^{2}\left(\frac{\partial u}{\partial z}-\frac{\partial w}{\partial x}\right)\right] \\
\frac{\partial^{2} w_{S}}{\partial t^{2}}=\frac{\partial}{\partial x}\left[V_{S}^{2}\left(\frac{\partial w}{\partial x}-\frac{\partial u}{\partial z}\right)\right] .
\end{gathered}
$$

Different from other methods of decoupled elastic-wave equations [Ma et al., 2003; Li et al., 2007; Tang and McMechan, 2018], there is no inhomogeneous-term in the new S-wave decoupled equation (Equation 23) and hence most $\delta V_{S} \rightarrow P P$ exist in the P-wavefield. Therefore the new decoupled equations are beneficial to improve the accuracy of the S-wavefield to reduce contamination on $K_{V_{S}}^{S S}$ from other kernels.

Next consider an inhomogeneous velocity model, where the velocity increases gradually along the radius and the minimum velocity value is at the center of the model. The $\mathrm{P}$ and S-waves are simulated using a Ricker source wavelet with peak frequency of $25 \mathrm{~Hz}$ and source location is at the center of the model. Snapshots of S-wavefield are shown in Figure 7. Figure 7a and $7 \mathrm{~b}$ are the $\mathrm{x}$-component and $\mathrm{z}$-component of S-wavefield containing the inhomogeneousterm, respectively; Figure $7 \mathrm{c}$ and $7 \mathrm{~d}$ are the $\mathrm{x}$-component and $\mathrm{z}$-component of S-wavefield without the inhomogeneous-term, respectively. Comparing Figure 7a, $7 \mathrm{~b}$ and Figure $7 \mathrm{c}, 7 \mathrm{~d}$, the superiority of the new decoupled method is to attenuate $\mathrm{P}$-wave components in the S-wavefield, through separation of $\delta V_{S} \rightarrow P P$ from the S-wavefield. Figure $7 \mathrm{e}$ shows the difference between Figure $7 \mathrm{a}$ and $7 \mathrm{c}$, and Figure $7 \mathrm{f}$ represents the difference between Figure $7 \mathrm{~b}$ and $7 \mathrm{~d}$. Obviously, the difference mostly comes from the P-wave component. Below, we call the elastic-wave decoupled method with inhomogeneous-term in the S-wave equation as "conventional decoupled method", and call the elastic-wave decoupled method without inhomogeneous-term in the S-wave equation as "our decoupled method".

To demonstrate the effectiveness of our decoupled ERFWI, we compare the two types of transmission kernels (gradient) with a single source-receiver pair in an inhomogeneous medium (the velocity increases with depth). The source and receiver positions are located on the top and bottom of the model, respectively. The P and S-waves are simulated using a Ricker source wavelet with peak frequency of $13 \mathrm{~Hz}$. As we can see from Figure 8, the conventional decoupled kernel (Figure 8a) produces more high-wavenumber artifacts than our decoupled method (Figure 8b). The difference (Figure 8c) between the two methods shows the high-wavenumber contamination due to the coupled effect from the P-wave.

\section{Numerical Example}

In the following section, we apply our decoupled method to a resampled Sigsbee2A model shown in Figure 9a and $9 \mathrm{~b}$. The dimension of the resampled model in the horizontal and vertical direction is $n x * n z=351 * 184$. The grid size is $10 \mathrm{~m}$ in both directions. In the initial model, velocity increases linearly with depth (Figure 9c and 9d). During the inversion, we keep the density constant $\left(2.0 \mathrm{~g} / \mathrm{cm}^{3}\right)$. The high-order staggered grid finite-difference scheme [Dong et al., 2000] serves as the wavefield extrapolator for forward modeling. The compressional source (explosive source) time wavelet is a Ricker wavelet with $13 \mathrm{~Hz}$ dominant frequency. There are 2500 time sampling points and the time sampling interval is $0.8 \mathrm{~ms}$. In total, 35 sources are exploded at a depth of $10 \mathrm{~m}$ with $100 \mathrm{~m}$ interval. The wavefields are recorded by 351 receivers covering the entire model at the surface for each shot. 


\section{Zhanyuan Liang et al.}
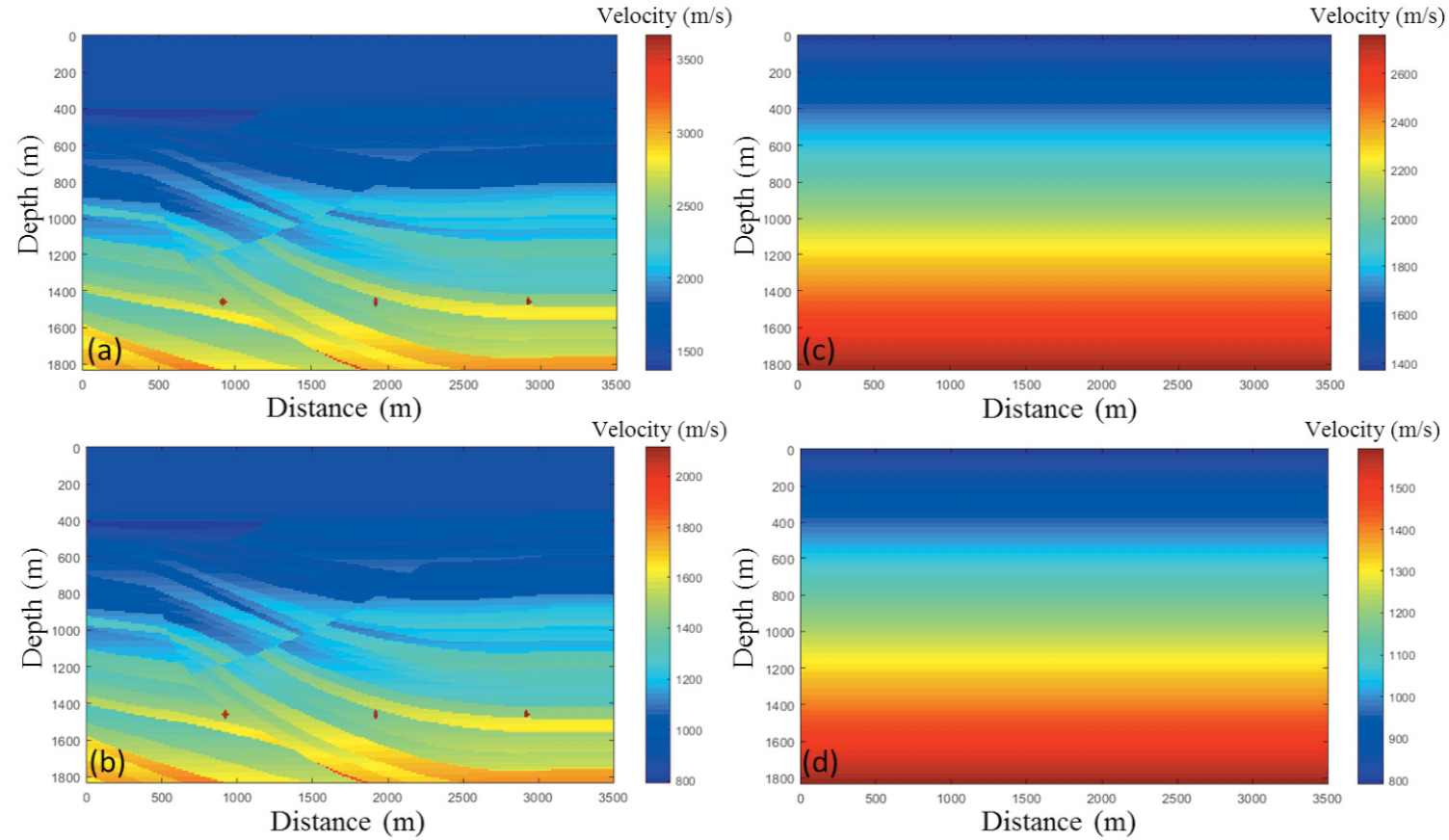

Figure 9. Sigsbee2A velocity model. (a) Sigsbee2A P-wave velocity model; (b) Sigsbee2A S-wave velocity model; (c) initial P-wave velocity model; (d) initial S-wave velocity model.

Let us further investigate the S-wave velocity gradient behavior for the Sigsbee2A model. We can take the linear initial velocity (Figure 9d) as a continuous inhomogeneous velocity. After decoupling the elastic-wave equation in inhomogeneous medium, we calculate the S-wave velocity gradient $K_{V_{S}}^{S S}$ with only the S-wavefield. Figure $10 \mathrm{a}$ and $10 \mathrm{~b}$
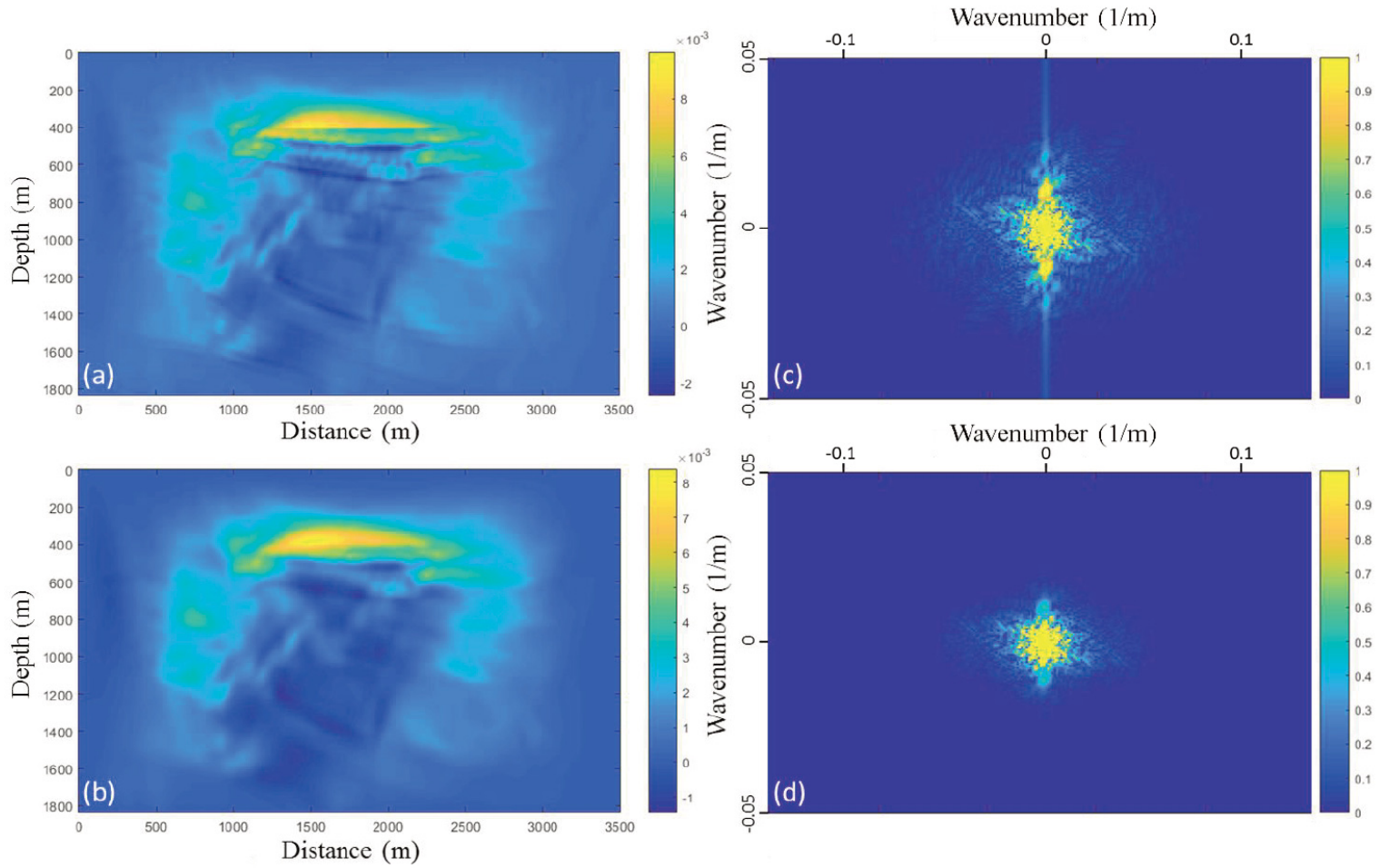

Figure 10. The S-wave velocity gradient of different methods and their wavenumber spectral distribution. (a) The S-wave velocity gradient of conventional decoupled ERFWI and (c) its wavenumber spectral distribution. (b) The S-wave velocity gradient of our decoupled ERFWI and (d) its wavenumber spectral distribution. 
show gradients for the conventional and our decoupled ERFWI, respectively. We can see that Figure 10b has more low-wavenumber information than the conventional decoupled ERFWI gradient (Figure 10a). To emphasize this aspect, we show the wavenumber spectral distribution of the two gradients, as shown in Figure 10c and 10d. It can be seen that the energy in Figure 10d focuses more on low-wavenumber components than that in Figure 10c. These tests suggest that our new decoupled ERFWI method is feasible and superior for updating the low-wavenumber components for the S-wave velocity.

Figure 11a shows the Sigsbee2A S-wave velocity model after resampling and its imaging result of reverse time migration (RTM) with cross-correlation imaging conditions (Figure 11d). Only when the initial velocity model is suited, the RTM image can be accurately focused. Figure 11e depicts the RTM images using the linear initial models (Figure 11b). Because the initial model is far away from the actual model, the travel-time information is inaccurate and the migration result is not focused, especially for the three diffraction points below the model. In addition, some deep reflectors are deeper than their true location, due to higher velocity value of the initial model. Figure11c and $11 \mathrm{f}$ show the updated low-wavenumber background models of S-wave velocity after 30 iterations (Figure 11c) and its migration result (Figure 11f) respectively. Accordingly, the RTM image, as illustrated by Figure 11f, is significantly improved with our decoupled ERFWI results. Besides, Figure 11f shows that the three diffraction points are better focused and are nearly the same as those in Figure 11d. Vertical velocity profiles from the different distance of the model are shown in Figure 12. The linear initial velocity (blue solid line) deviates from the true one (black solid line), and after our decoupled ERFWI, the trend of the inverted velocity curve (red solid line) is closer to the true curve.
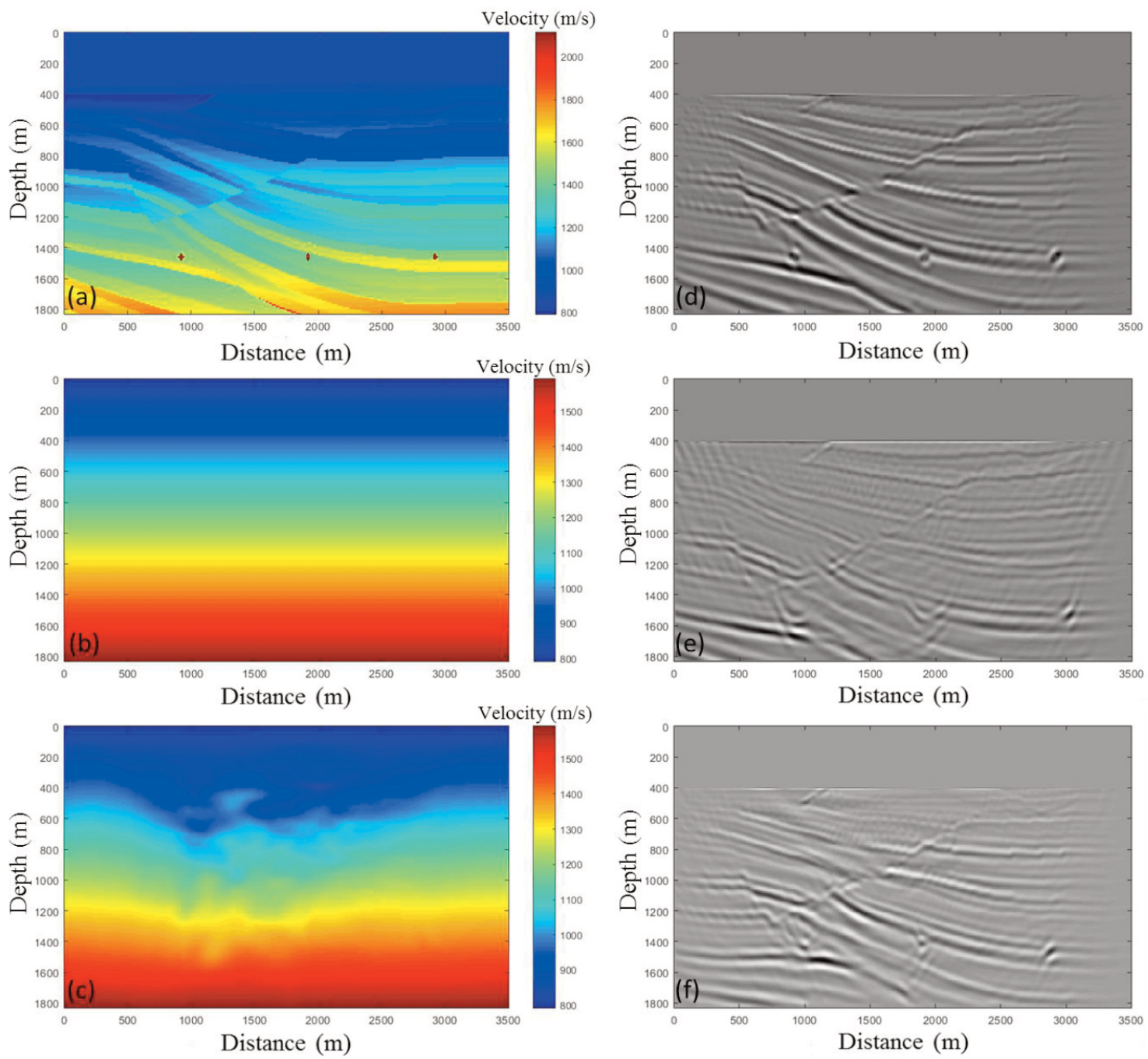

Figure 11. Sigsbee2A S-wave velocity model after resampling (a) and its image result of reverse time migration (RTM) (d), initial Swave velocity model (b) and its image result of RTM (e), inverted S-wave velocity after 30 iterations with our decoupled ERFWI and its image result of RTM. 


\section{Zhanyuan Liang et al.}

To further prove the ability of our new method in updating low-wavenumber velocity, we perform elastic-wave full waveform inversion (EFWI) by taking the inverted velocity by our decoupled ERFWI as initial velocity. All the parameters of numerical simulation are the same as the RFWI above. Inverted velocities after 96 iterations with EFWI are shown as Figure 13c (the inverted P-wave velocity) and 13d (the inverted S-wave velocity). After normalizing, the convergence curve of errors in the process of EFWI is shown in Figure 14. From Figure 14, we can see that the convergence of errors is fast and stable without falling into the local minimum, which shows the accuracy of the low-wavenumber velocity obtained by our decoupled ERFWI. Vertical velocity profiles from the different distance of the P-wave velocity and the S-wave velocity are shown in Figure 15 and Figure 16, respectively. After EFWI, we can get the ideal velocity profiles.
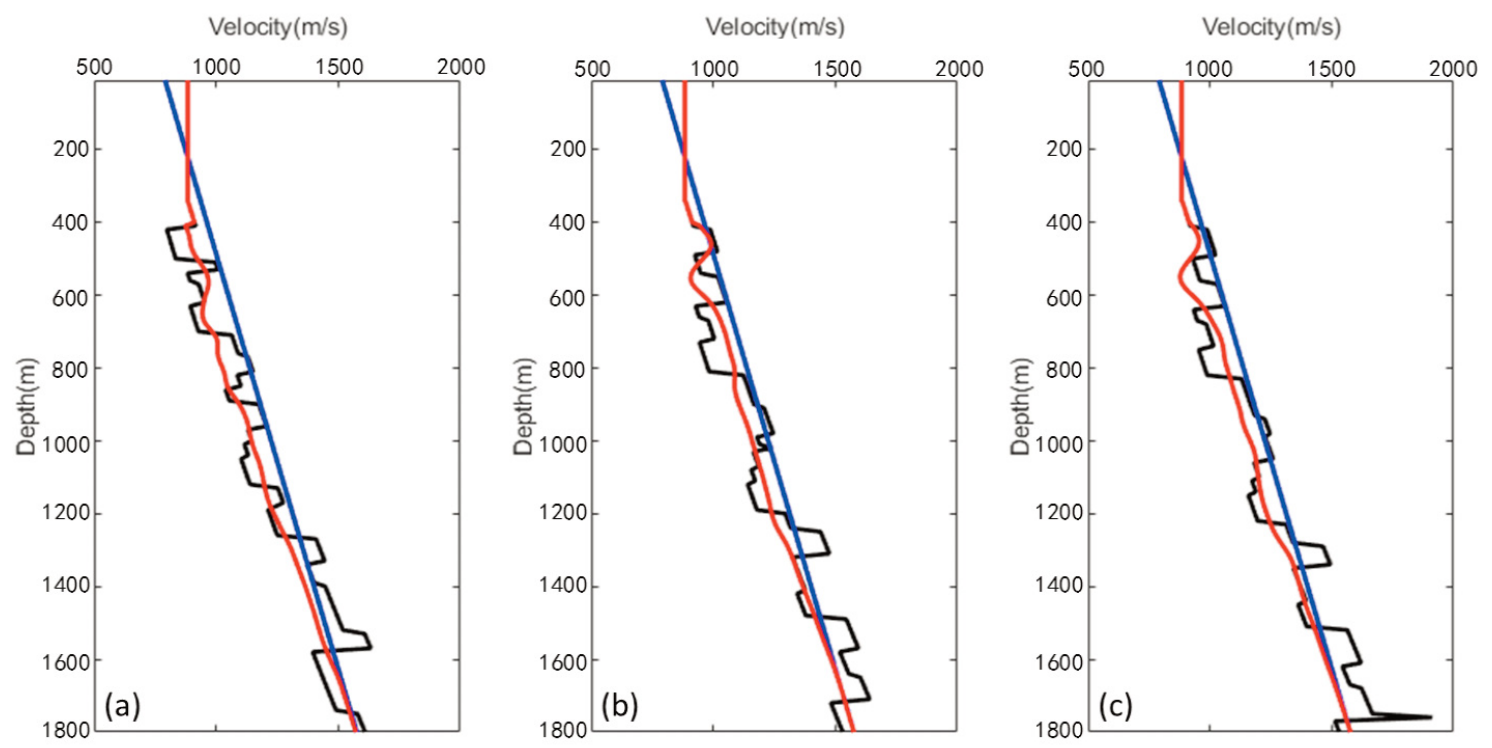

Figure 12. Vertical velocity profiles of the Sigsbee2A S-wave velocity (black solid line), the linear initial S-wave velocity (blue solid line) and the inverted S-wave velocity after 30 iterations of our decoupled ERFWI (red solid line) with different distance (a) $1000 \mathrm{~m}$, (b) $1500 \mathrm{~m}$, (c) $1600 \mathrm{~m}$.

\section{Conclusions}

A good initial velocity model with accurate low-wavenumber components is the key factor for FWI. According to the equation $\mathbf{k}=\left(2 f / c_{0}\right) \cos (\theta / 2) \mathbf{n}$, the update of low-wavenumber velocity mainly depends on low-frequency information in the seismic data and tomographic components in gradients (cross-correlation of wavefield with scattering angle close to $180^{\circ}$ ). However in reality, there is little low-frequency information below $5 \mathrm{~Hz}$ in the seismic data. To address this problem, in this paper, we construct the initial velocity model through RFWI method that is dependent on tomographic components, which is more in line with the physical meaning between wave propagation and velocity imaging.

The parameter of the acoustic method is only P-wave velocity with PP scattered wavefield. However, not only PP scattered waves but also PS converted waves exist in the elastic wavefield (Figure 3), there will be the issue of crosstalk between the inverted P-wave velocity and inverted S-wave velocity (Figure 4). In particular, the inversion of S-wave velocity is more susceptible to the coupling effects of elastic wavefield than P-wave velocity. To overcome that, we calculate the S-wave velocity with only S-wavefield according to the theory of elastic-wave equations decoupling [Wang et al., 2018b] which is beneficial to reduce the P-wave components of S-wavefield. This requires a sufficiently high-precision S-wavefield, but in inhomogeneous medium, the decoupled S-wavefield is still subject to strong P-wave components. Hence, we further derive a set of equations suitable for the decoupling in an inhomogeneous medium to reduce the coupling effects of P-wavefield on S-wavefield. The numerical results of Sigsbee2A model demonstrate that our decoupled ERFWI can provide an accurate initial velocity model for FWI. 

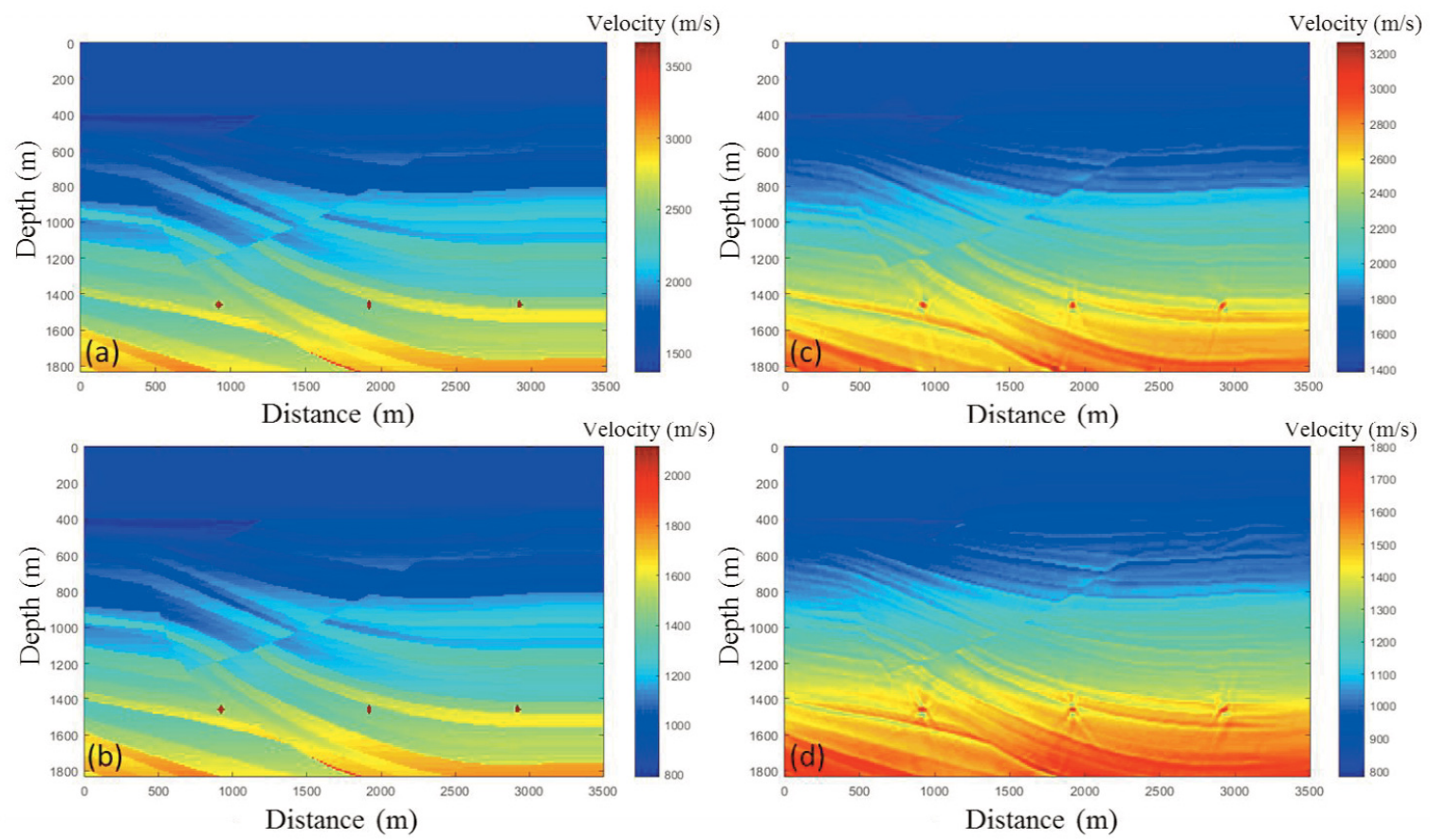

Figure 13. Inverted velocity with elastic full waveform inversion (EFWI). (a) Sigsbee2A P-wave velocity after resampling; (b) Sigsbee2A S-wave velocity after resampling; (c) inverted P-wave velocity after 96 iterations with EFWI; (d) inverted Swave velocity after 96 iterations with EFWI.

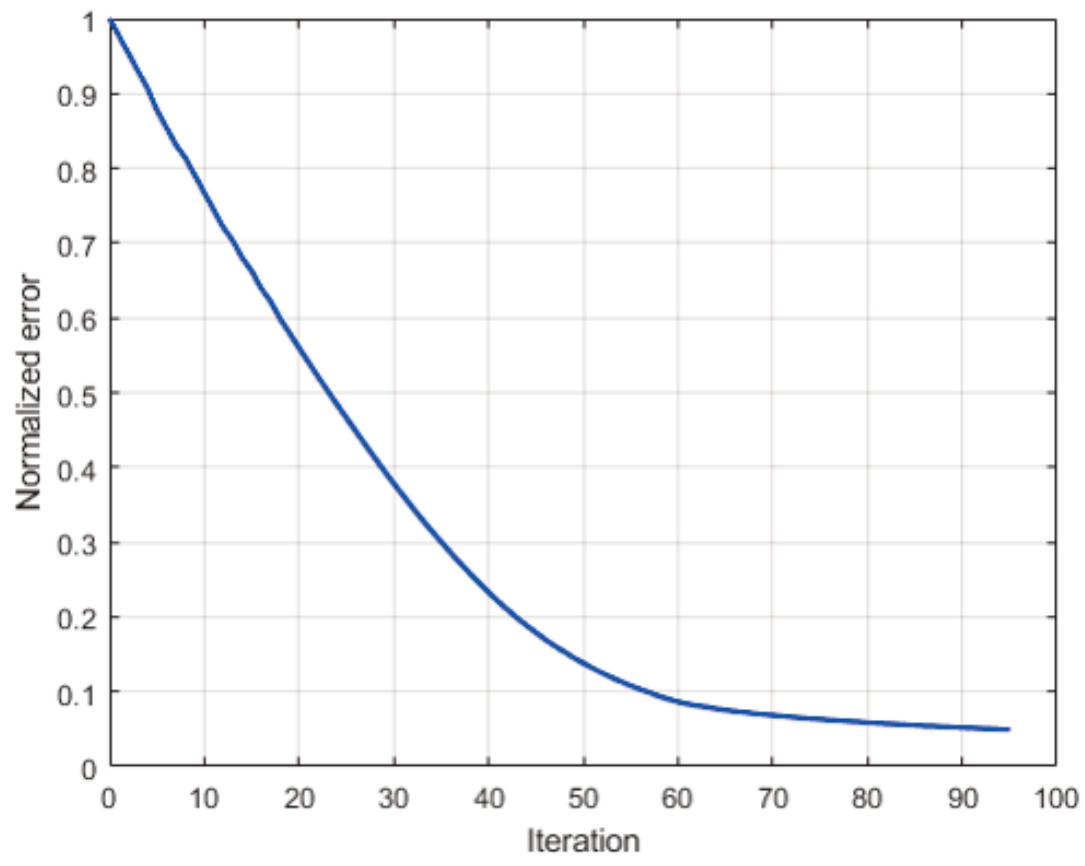

Figure 14. The convergence curve of errors in the EFWI. 


\section{Zhanyuan Liang et al.}
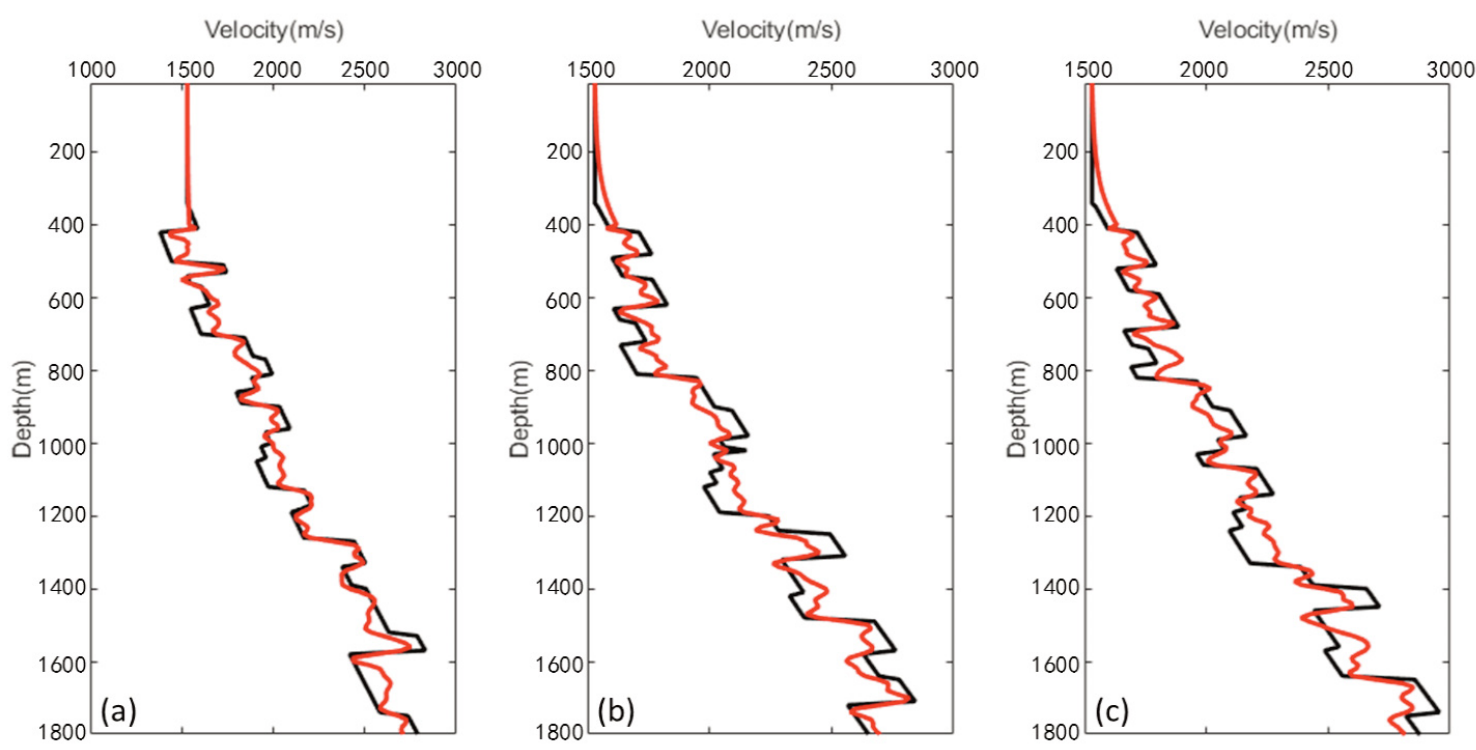

Figure 15. Vertical velocity profiles of the Sigsbee2A P-wave velocity (black solid line) and the inverted P-wave velocity after 96 iterations of EFWI (red solid line) with different distance (a) $1000 \mathrm{~m}$, (b) $1500 \mathrm{~m}$, (c) $1600 \mathrm{~m}$.
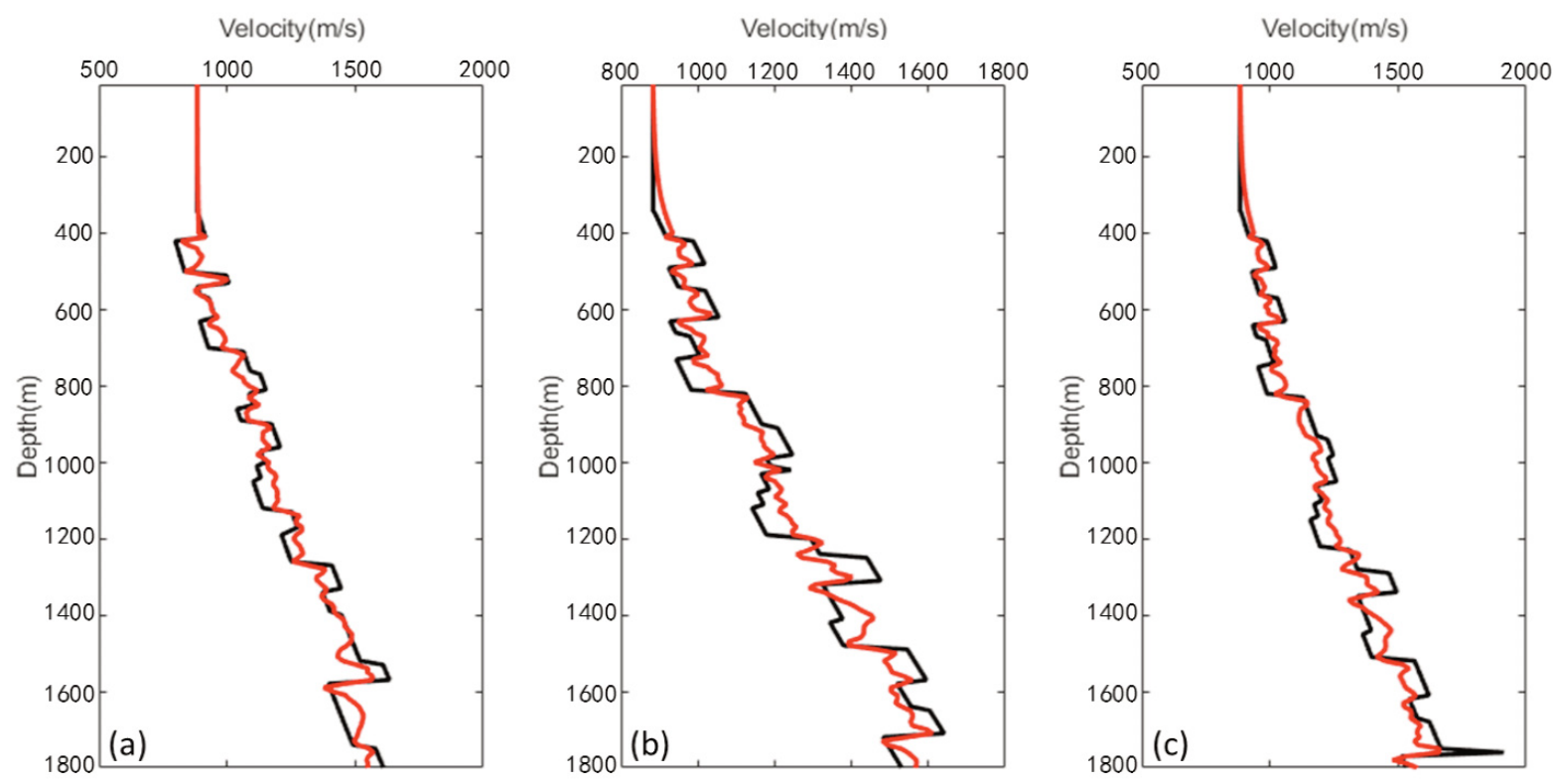

Figure 16. Vertical velocity profiles of the Sigsbee2A S-wave velocity (black solid line) and the inverted S-wave velocity after 96 iterations of EFWI (red solid line) with different distance (a) 1000m, (b) 1500m, (c) 1600m.

Acknowledgement. Thanks to the reviewers for their valuable comments and thanks to the editor for their careful profreading and revision. I would like to thank the support of National Science and Technology Major Sub-project "MiddleDeep Complex Reservoir Characterization and Oil and Gas Detection Technology Based on Wide-Azimuth Prestack Seismic Inversion" (2016ZX05024-001-008) and the National Natural Science Foundation of China Joint Fund Project "Unconventional Oil and Gas Enrichment Mechanism" Joint funding with Geophysical Dessert Identification (U1562215) and "the Fundamental Research Funds for the Central Universities" (17CX06045). 


\section{Decoupled ERWI in Inhomogeneous Medium}

\section{References}

Baeten G., J.W. De Maag and R. E. Plessix (2013). The use of low frequencies in a full waveform inversion and impedance inversion land seismic case study, Geophys. Prospect., 61, 701-711.

Baysal E., D. Kosloff and J. Sherwood (1983). Reverse time migration, Geophys., 48, 1514-1524.

Bharadwaj P., W. Mulder and G. Drijkoningen (2016). Full waveform inversion with an auxiliary bump functional, Geophys. J. Int., 206, 1076-1092.

Biondi B. and A. Almomin (2013a). Tomographic FWI (TFWI) by extending the velocity model along the time-lag axis, In Proceedings of the SEG Technical Program, Expanded Abstracts, 1031-1034.

Biondi B. and A. Almomin (2013b). Tomographic full-waveform inversion (TFWI) by combining FWI and waveequation migration velocity analysis, The Leading Edge, 32, 1074-1080.

Biondi B. and W. Symes (2004). Angle-domain common-image gathers for migration velocity analysis by wavefieldcontinuation imaging, Geophys., 69, 1283-1298.

Bunks C., F.M. Salek S. Zaleski and G. Chavent (1995). Multiscale seismic waveform inversion, Geophys., 60, 14571473.

Chang W.F. and G.A. McMechan (1987). Elastic reverse-time migration, Geophys., 52, 1365-1375.

Cheng J., T. Alkhalifah, Z. Wu, P. Zou and C. Wang (2016). Simulating propagation of decoupled elastic waves using low-rank approximate mixed-domain integral operators for anisotropic media, Geophys., 81, T63-T77.

Chi B., L. Dong and Y. Liu (2015). Correlation-based reflection full-waveform inversion, Geophysics, 80, R189-R202.

Claerbout, J. and S. Doherty (1972). Downward continuation of moveout corrected seismograms, Geophys., 37, 741768.

Clayton, R.W. (1981). Wavefield inversion methods for refraction and reflection data, Ph.D. thesis, Stanford University.

Crase E., A. Picat, M. Noble, J. McDonalds and A. Tarantola (1990). Robust elastic nonlinear waveform inversion: Application to real data, Geophys., 55, 527-538.

Díaz E., and P. Sava (2014). Wavefield tomography using reverse time migration backscattering, Geophys., 80, R57R69.

Djebbi R., and T. Alkhalifah (2014). Traveltime sensitivity kernels for wave equation tomography using the unwrapped phase, Geophys. J. Int., 197, 975-986.

Du Q., C.F. Guo, Q. Zhao, X. Gong, C. Wang and X. Li (2017). Vector-based elastic reverse time migration based on scalar imaging condition, Geophys., 82, S111-S127.

Du Q., X. Gong, M. Zhang, Y. Zhu and G. Fang (2014). 3D PS-wave imaging with elastic reverse-time migration, Geophys., 79, S173-S184.

Du Q., Y. Zhu and J. Ba (2012). Polarity reversal correction for elastic reverse time migration, Geophys., 77, S31-S41.

Elita L.Y., Y. Du, J. Yang, A. Cheng, X. Fang (2018). Elastic reverse time migration using acoustic propagators. Geophysics, 83, 1-37.

Gazdag J. (1978). Wave equation migration with the phase-shift method, Geophys., 43, 1342-1351.

Hardy P. (2013). Ongoing R\&D in ray based tomography: well worth the effort, $83^{\text {rd }}$ Annual International Meeting, SEG, Expanded Abstracts, 4806-4810.

$\mathrm{Hu}$ W. (2014). FWI without low frequency data-beat tone inversion, $84^{\text {th }}$ Annual International Meeting, SEG, Expanded Abstracts, 1116-1120.

Jannane M., W. Beydoun, E. Crase, D. Cao, Z. Koren, E. Landa, M. Mendes, A. Pica, M. Noble, G. Roeth, S. Singh, R. Snieder, A. Tarantola, D. Trezeguet, M. Xie (1989). Wavelengths of earth structures that can be resolved from seismic reflection data, Geophys., 54, 906-910.

Lailly P. (1983). The seismic inverse problem as a sequence of before stack migrations. Conference on Inverse Scattering, Theory and Application, Society for Industrial and Applied Mathematics, Expanded Abstracts, 206-220.

Li Z., H. Zhang, Q. Liu and W. Han (2007). Numeric simulation of elastic wavefield separation by staggering grid high-order finite-difference algorithm (in Chinese): Oil Geophys. Prospect., 42, 510-515.

Ma D. and G. Zhu (2003). P- and S-wave separated elastic wave equation numerical modeling (in Chinese), Oil Geophys. Prospect., 38, 482-486.

Mora P. (1989). Inversion = migration + tomography, Geophys., 54, 1575-1586. 


\section{Zhanyuan Liang et al.}

Mora P.R. (1987). Nonlinear two-dimensional elastic inversion of multi-offset seismic data, Geophys., 52, 12111228.

Morse P.M. and H. Feshbach (1954). Methods of theoretical physics, Am. J. Phys., 22, 410-413.

Shabelansky A.H., A.E. Malcolm, M.C. Fehler, X. Shang and W. L. Rodi (2015). Source-independent full wavefield converted-phase elastic migration velocity analysis, Geophys. J. Int., 200, 954-968.

Shin C., and Y. H. Cha (2008). Waveform inversion in the Laplace domain, Geophys. J. Int., 173, 922-931.

Sirgue L., and R.G. Pratt (2004). Efficient waveform inversion and imaging: A strategy for selecting temporal frequencies, Geophys., 69, 231-248.

Stolt R.H. (1978). Migration by Fourier transform, Geophys., 43, 23-48.

Sun R., G.A. McMechan and H. Chuang (2011). Amplitude balancing in separating P and S waves in 2D and 3D elastic seismic data, Geophys., 76, S103-S113.

Sun R., Chow J. and K.J. Chen (2001). Phase correction in separating p-and s-waves in elastic data, Geophys., 66, 1515-1518.

Symes W.W., and M. Kern (1994). Inversion of reflection seismograms by differential semblance analysis: Algorithm structure and synthetic examples, Geophys. Prospect., 42, 565-614.

Tang C., and GA. McMechan (2018). Multidirectional-vector-based elastic reverse time migration and angle-domain common-image gathers with approximate wavefield decomposition of P-and S-waves, Geophys., 83, S57-S79.

Tang Y., S. Lee, A. Baumstein, D. Hinkley (2013). Tomographically enhanced full wavefield inversion, 2013 SEG Annual Meeting, Society of Exploration Geophys., 1037-1041.

Tarantola A. (1984). Inversion of seismic reflection data in the acoustic approximation, Geophys., 49, 1259-1266.

Tarantola A. (1986). A strategy for nonlinear elastic inversion of seismic reflection data, Geophys., 51, $1893-1903$.

Virieux J. and S. Operto (2009). An overview of full-waveform inversion in exploration geophysics, Geophys., 74, WCC1-WCC26.

Wang G.C., S.X. Wang, Q.Z. Du and S.Y. Yuan (2017). Traveltime-based reflection full-waveform inversion for elastic medium, J. Applied Geophys., 141, 68-76.

Wang G, Wang S, Song J., C. Dong, M. Zhang (2018a). Elastic reflection traveltime inversion with decoupled wave equation, Geophys., 83, R463-R474.

Wang T, J. Cheng J., Q. Guo and C. Wang (2018b). Elastic wave-equation-based reflection kernel analysis and traveltime inversion using wave mode decomposition, Geophys, J. Int., 215, 450-470.

Wang W., and G.A. McMechan (2015). Vector-based elastic reverse time migration, Geophys., 80, S245-S258.

Wu R.S., J. Luo and B. Wu (2014). Seismic envelope inversion and modulation signal model, Geophys., 79, WA13WA24.

Xiao X., and W.S Leaney (2010). Local vertical seismic profiling (VSP) elastic reverse-time migration and migration resolution: Salt-flank imaging with transmitted P-to-S waves, Geophys., 75, S35-S49.

Xu S., D. Wang, F. Chen, G. Lambare and Y. Zhang (2012a). Inversion on reflected seismic wave, 82nd Annual International Meeting, SEG, Expanded Abstracts.

Xu S., D. Wang, F. Chen, Y. Zhang and G. Lambare (2012b). Full waveform inversion for reflected seismic data, 74th Annual International Conference and Exhibition, EAGE, Extended Abstracts.

Yilmaz O. (2001). Seismic data analysis: processing, inversion and interpretation of seismic data, Society of Exploration Geophysicsts. 